\title{
Reporting quality of trend analyses in leading medicine and oncology journals
}

Xiaoling Yuan, $\mathrm{MD},{ }^{1}$ Yong Lin, $\mathrm{PhD},{ }^{2}$ Yating Wang, $\mathrm{MD},{ }^{3,}{ }^{*}$ Lanjing Zhang, $\mathrm{MD}^{4 *}$

${ }^{1}$ Department of Infectious Disease, Ninth People's Hospital, Shanghai Jiao Tong University School of Medicine, Shanghai, China; ${ }^{2}$ Department of Biostatistics and Epidemiology, Rutgers University School of Public Health, Piscataway, New Jersey; ${ }^{3}$ Department of Medicine, Weiss Medical Center, Chicago, Illinois, USA; ${ }^{4}$ Department of Biological Sciences, Rutgers University, Newark, New Jersey, USA

Correspondence: Lanjing Zhang, MD, Department of Pathology, Princeton Medical Center, 1 Plainsboro Rd., Plainsboro, NJ 08563. Email: lanjing.zhang@rutgers.edu, Yating Wang, MD, Department of Medicine, Weiss Medical Center, Chicago, Illinois, USA, Email:

yatingwang91@gmail.com

*Equal contribution.

Running head: Reporting quality of trend analyses

Word count: 600

Key words: Trend, reporting quality, medical journal, oncology journal 


\section{Abstract}

Trend analyses are critical for assessing changes and predicting future of epidemiological parameters. Recent trend-analysis guidelines recommended reporting slopes or beta/coefficient if possible. American Statistical Association and others recommended reporting effect-size. However, reporting of these statistics in trend analyses was largely unclear. We searched the Pubmed for the articles published during Jan. 1, 2008 to Dec. 31, 2018, whose titles included "trend" or "trends" among the following medicine and oncology journals: Ann Intern Med, Ann Oncol, BMJ, J Clin Oncol, J Natl Cancer Inst, JAMA Oncol, JAMA, Lancet, Lancet Oncol and N Engl J Med. We examined proportions of the trend analyses in leading medicine and oncology journals, that reported p-value, effect-size or beta/coefficient/Annual percent change (APC), and identified associated factors. Among the 398 identified reports of trend analysis published during 2008-2018, there were 297 qualified reports, including 38 (12.8\%) analyses using nonparametric model, 226 (76.1\%) analyses using (piece-wise) linear model, 32 (10.8\%) analyses using non-linear parametric model and $1(0.3 \%)$ analyses using semi-parametric model (Cox regression). In these analyses, $193(66.0 \%)$ and $216(72,7 \%)$ analyses reported $P$-value and effect-size, respectively. Among the 226 trend analyses using linear model, 169 (74.8\%) reported p-value, $183(81.0 \%)$ reported effect-size, 94 (41.6\%) reported APC and 34 (15.0\%) reported Beta/coefficient. Only 13 (5.8\%) analyses reported neither $p$-value/effect size nor beta/coefficient/APC. In multivariable regression models, author affiliation with epidemiology department or statistics department was associated with reporting effect-size, and U.S. seniorauthors (versus non-U.S.) more likely reported $p$-value. No factors were linked to reporting APC. In summary, reporting quality of the included trend analyses was overall good. However, reporting $p$-value or effect-size did not change with publication years, and reporting APC or 
bioRxiv preprint doi: https://doi.org/10.1101/2020.09.18.303701; this version posted September 21, 2020. The copyright holder for this preprint (which was not certified by peer review) is the author/funder. All rights reserved. No reuse allowed without permission.

Beta/coefficient was uncommon. Additional works and training appear warranted to improve reporting quality of trend analyses. 
Trend analyses are critical for assessing changes and predicting future of epidemiological parameters. ${ }^{1,2}$ Recent trend-analysis guidelines recommended reporting slopes or beta/coefficient if possible. ${ }^{3}$ American Statistical Association and others recommended reporting effect-size. ${ }^{4,5}$ However, reporting of these statistics in trend analyses was largely unclear. We therefore examined proportions of the trend analyses in leading medicine and oncology journals, that reported p-value, effect-size or beta/coefficient/Annual percent change (APC), and identified associated factors.

Methods | We searched the Pubmed for the articles published during Jan. 1, 2008 to Dec. 31, 2018, whose titles included "trend" or "trends" among the following medicine and oncology journals: Ann Intern Med, Ann Oncol, BMJ, J Clin Oncol, J Natl Cancer Inst, JAMA Oncol, JAMA, Lancet, Lancet Oncol and N Engl J Med. We only included original articles, research letters and meta-analyses/systematic reviews of trend analysis. Three of the authors independently reviewed full-texts of the articles to extract: publication year, journal specialty (medicine/oncology), model type, reporting of $P$-value, effect-size (defined as quartiles/confidence/credible/uncertainty intervals) and co-efficient/slope/Annual Percent Change (APC), senior author(s)' location, and any author in School of Public Health, statistics department, or epidemiology department.

According to guidelines, ${ }^{3}$ co-efficient/slope/APC should be reported in (piece-wise) linear models. We assessed reporting whether co-efficient/slope/APC was reported in the analyses using linear model. We also scored the reporting quality of these analyses by assigning 1 point for reporting $\mathrm{p}$-value or effect-size, and another point for reporting beta/co-efficient/slope/APC. 
The sum of each article's scores was considered as its reporting-quality score, which was up to 2 points.

We used Chi-square test, Fisher exact test, and (ordinal) logistic regression to examine potential associations (Stata, version 15). Only the factors with $\mathrm{P}<0.10$ shown in univariate analysis were included in multivariable logistic regression analyses. Two-sided P values were reported. Statistical significance was considered when $\mathrm{P}<0.05$.

Results | Among the 398 identified reports of trend analysis published during 2008-2018, there were 297 qualified reports (SFigure 1), including 38 (12.8\%) analyses using non-parametric model, 226 (76.1\%) analyses using (piece-wise) linear model, 32 (10.8\%) analyses using nonlinear parametric model and $1(0.3 \%)$ analyses using semi-parametric model (Cox regression). Among these analyses, $193(66.0 \%)$ and 216 (72,7\%) analyses reported $P$-value and effect-size, respectively. Subgroup analyses showed that U.S. senior authors more likely reported $P$-value or effect-size than non-U.S. senior authors (Figure), while reporting of these parameters was not linked to other factors.

Among the 226 trend analyses using linear model (STable 1), 169 (74.8\%) reported p-value, 183 (81.0\%) reported effect-size, 94 (41.6\%) reported APC and 34 (15.0\%) reported Beta/coefficient. Only 13 (5.8\%) analyses reported neither $p$-value/effect size nor beta/coefficient/APC. Ordinal logistic regression showed only author affiliation with school of public health was linked to higher reporting-quality scores (Odds ratio=7.44, 95\% confidence interval, $3.22-31.17$ ). In multivariable regression models, author affiliation with epidemiology department or statistics 
department was associated with reporting effect-size, and U.S. senior-authors (versus non-U.S.) more likely reported p-value. No factors were linked to reporting APC (STable 2).

Discussion | Reporting quality of the included trend analyses was overall good. However, several issues are concerning. Reporting $p$-value or effect-size did not change with publication years, despite the recommendation on using effect-size. ${ }^{4,5}$ Moreover, Non-U.S. senior authors less frequently reported p-value or effect-size than U.S. ones, which warrants more research and training. Furthermore, more than half of the trend analyses using linear model did not report p-value/effect-size, slope/beta/APC or either, which is not consistent with recommendations. We thus call for more research and awareness of reporting-quality in trend analyses.

Limitations included possible misclassification of reporting data and inclusion of only leading journals. The used reporting-quality metrics may not be applicable to all reports.

Author Contributions: Drs Zhang and Wang had full access to all of the data in the study and takes responsibility for the integrity of the data and the accuracy of the data analysis. They equally supervised the works, and share the senior authorship.

Concept and design: Yuan, Wang, Zhang.

Acquisition, analysis, or interpretation of data: All authors.

Drafting of the manuscript: Yuan.

Critical revision of the manuscript for important intellectual content: All authors. 
Statistical analysis: Yuan, Lin, Zhang.

Supervision: Zhang, Wang.

\section{Figure Legend}

Proportions of the trend analyses published during 2008-2018 reporting $p$-value and effect-size and their associated factors.

Only senior author's affiliation (U.S.A. vs non U.S.A) was linked to reporting either p-value (A) or effect size (B) among the 397 trend analyses published in leading medicine and oncology journals during 2008-2018.

\section{Bibliography}

1. Zhang J, Lin Y, Zhang L. Trends in Alcoholic Fatty Liver Disease. JAMA 2019;322:979-80. 2. Wong T, Dang K, Ladhani S, Singal AK, Wong RJ. Prevalence of Alcoholic Fatty Liver Disease Among Adults in the United States, 2001-2016. JAMA 2019;321:1723-5.

3. Ingram DD, Malec DJ, Makuc DM, et al. National Center for Health Statistics Guidelines for Analysis of Trends. Vital Health Stat 2 2018:1-71.

4. Wasserstein RL, Lazar NA. The ASA statement on p-values: context, process, and purpose. Taylor \& Francis; 2016.

5. Harrington D, D'Agostino RB, Sr., Gatsonis C, et al. New Guidelines for Statistical Reporting in the Journal. N Engl J Med 2019;381:285-6. 


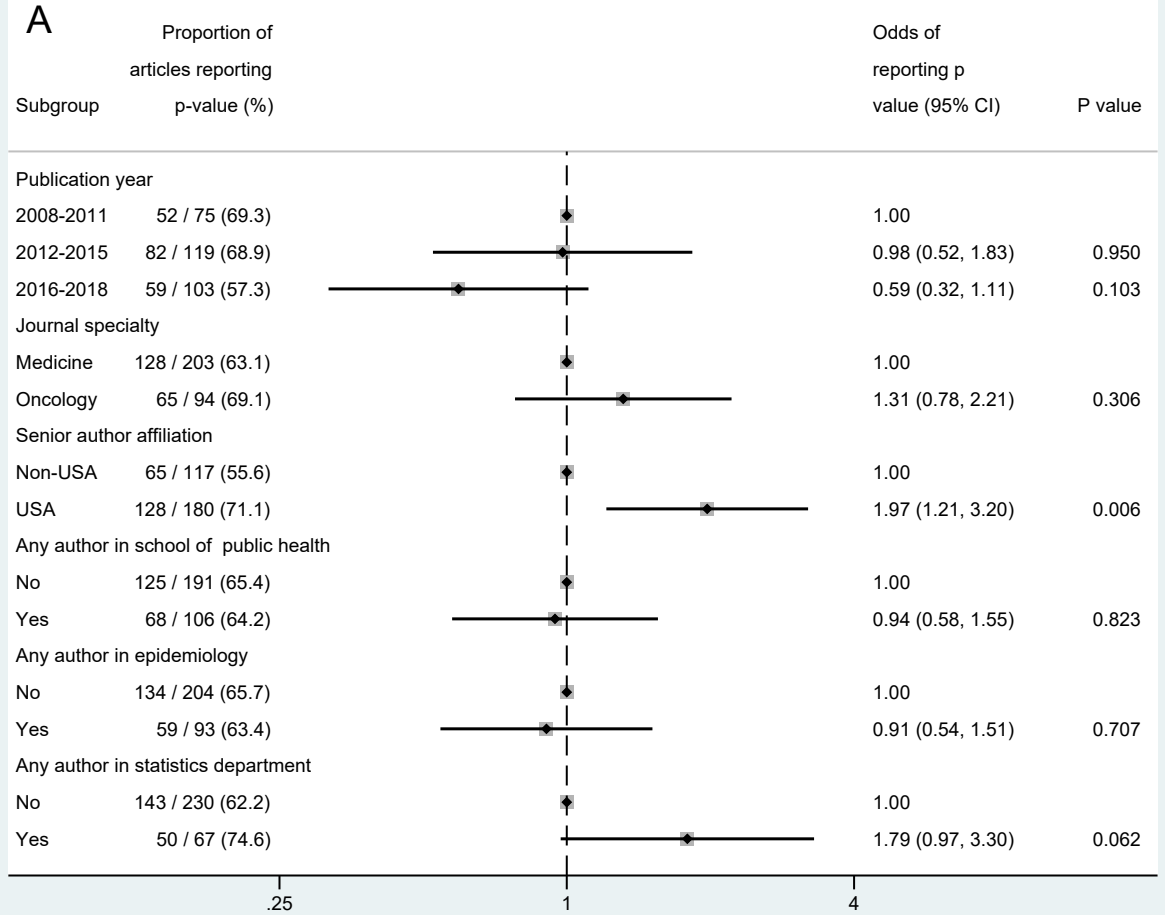




\section{B}

Proportion of

articles reporting

Subgroup effect-size (\%)
Odds of reporting

effect-size

$(95 \% \mathrm{Cl})$

$P$ value
Publication year

2008-2011 $51 / 75$ (68.0)

2012-2015 $89 / 119$ (74.8)

2016-2018 $76 / 103$ (73.8)

Journal specialty

Medicine $\quad 147 / 203(72.4)$

Oncology $\quad 69 / 94$ (73.4)

Senior author affiliation

Non-USA $74 / 117$ (63.2)

USA $\quad 142 / 180(78.9)$

Any author in school of public health

No

$136 / 191$ (71.2)

Yes

$80 / 106$ (75.5)

Any author in epidemiology department

No

152 / 204 (74.5)

Yes

64 / 93 (68.8)

Any author in statistics department

No

$162 / 230$ (70.4)

Yes

54 / 67 (80.6)

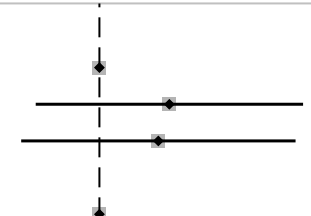

1.00

$1.40(0.74,2.64) \quad 0.305$

$1.32(0.69,2.55)$

0.400

1.00

$1.05(0.61,1.82)$

0.859

1.00

$2.17(1.29,3.65)$

0.003

1.00

$1.24(0.72,2.14)$

0.429

1.00

$0.75(0.44,1.30)$

0.308

1.00

$1.74(0.89,3.40)$

0.103 